\title{
Origin of the Magnetoresistance in Oxide Tunnel Junctions Determined through Electric Polarization Control of the Interface
}

\author{
Hisashi Inoue, ${ }^{1}$ Adrian G. Swartz, ${ }^{1, *}$ Nicholas J. Harmon, ${ }^{2}$ Takashi Tachikawa, ${ }^{3,4}$ Yasuyuki Hikita, ${ }^{3}$ \\ Michael E. Flatté, ${ }^{2}$ and Harold Y. Hwang ${ }^{1,3}$ \\ ${ }^{1}$ Department of Applied Physics, Geballe Laboratory for Advanced Materials, Stanford University, \\ Stanford, California 94305, USA \\ ${ }^{2}$ Department of Physics and Astronomy and Optical Science and Technology Center, \\ University of Iowa, Iowa City, Iowa 52242, USA \\ ${ }^{3}$ Stanford Institute for Materials and Energy Sciences, SLAC National Accelerator Laboratory, \\ Menlo Park, California 94025, USA \\ ${ }^{4}$ Department of Advanced Materials Science, The University of Tokyo, Kashiwa, Chiba 277-8561, Japan
}

(Received 23 September 2015; published 11 November 2015)

\begin{abstract}
The observed magnetoresistance (MR) in three-terminal (3T) ferromagnet-nonmagnet (FM-NM) tunnel junctions has historically been assigned to ensemble dephasing (Hanle effect) of a spin accumulation, thus offering a powerful approach for characterizing the spin lifetime of candidate materials for spintronics applications. However, due to crucial discrepancies of the extracted spin parameters with known materials properties, this interpretation has come under intense scrutiny. By employing epitaxial artificial dipoles as the tunnel barrier in oxide heterostructures, the band alignments between the FM and NM channels can be controllably engineered, providing an experimental platform for testing the predictions of the various spin accumulation models. Using this approach, we have been able to definitively rule out spin accumulation as the origin of the 3T MR. Instead, we assign the origin of the magnetoresistance to spin-dependent hopping through defect states in the barrier, a fundamental phenomenon seen across diverse systems. A theoretical framework is developed that can account for the signal amplitude, linewidth, and anisotropy.
\end{abstract}

DOI: 10.1103/PhysRevX.5.041023

\section{INTRODUCTION}

Three-terminal magnetoresistance (3T MR) in ferromagnet-nonmagnet (FM-NM) tunnel junctions has developed into an important phase-sensitive characterization tool for the rapid identification of materials that can transport spin information over long distances, a crucial feature for spintronic devices [1-5]. In principle, spin injection from a FM electrode and detection of the resulting spin accumulation in a NM semiconductor can be achieved using a single FM electrode in a FM-I-NM structure [1,5], as first demonstrated in $n$-GaAs [6]. This spin accumulation in the NM material generates a small spin-dependent voltage across the junction, which can be removed by Hanle dephasing under an applied magnetic field, producing junction MR with a Lorentzian line shape. Since the observation of such signals at room temperature in $\mathrm{Si}$ -

\footnotetext{
*Corresponding author. aswartz@stanford.edu

Published by the American Physical Society under the terms of the Creative Commons Attribution 3.0 License. Further distribution of this work must maintain attribution to the author(s) and the published article's title, journal citation, and DOI.
}

Subject Areas: Materials Science,

Semiconductor Physics,

Spintronics

based junctions [1], many groups have observed unexpectedly large Lorentzian MR in FM-I-NM heterojunctions, seemingly independent of many known materials parameters and spin-dephasing behavior, generating much debate over the origin of the observed phenomena [5,7-12]. As an example, long spin-coherence times have been inferred in $p$-type semiconductors despite the known rapid dephasing in the valence band [13].

Given these inconsistencies, it has been proposed that the MR might arise from spin accumulation in interfacial localized states (ILS), which could possibly generate the enhanced signals observed in experiments [2,14]. In this scenario, a spin splitting of the electrochemical potential of a system of localized states near the interface can generate spin accumulation, if this splitting persists in the limit of weak coupling of these states to the electrode and each other. Without viable alternatives, this scenario has often been accepted at face value to explain the observation of Lorentzian MR observed across many different materials systems. However, there are few experiments which can definitively support or refute a Hanle interpretation (in either the semiconductor or in ILS) [8], leaving the origin of the magnetoresistance in question and reducing its potential for identifying new spin-transport materials. 
The development of epitaxial complex oxide heterostructures enables the integration of various functional materials [15] offering a promising approach for realizing spintronics devices where oxide properties, such as spontaneous polarizations controllable by strain or applied voltages, can manipulate the electron spin degree of freedom. Combining such dramatic effects with $\mathrm{SrTiO}_{3}$, a semiconductor with high mobilities $[16,17]$ and potentially long spin lifetimes [18], could lead to novel spintronic devices. To date, the primary experimental evidence for spin injection into $\mathrm{SrTiO}_{3}$ is through the 3T approach, interpreted in terms of spin accumulation in ILS $[3,4]$ (or the $\mathrm{SrTiO}_{3}$ itself [19]), similar to $\mathrm{Si}$ and GaAs $[2,5,13]$. The epitaxial $\mathrm{LaAlO}_{3} / \mathrm{SrTiO}_{3}(001)$ heterostructure is one system with tunable interface properties, arising from the stacking of $\mathrm{AlO}_{2}^{-}$and $\mathrm{LaO}^{+}$charged layers. A consequence of this internal electric field is manifest in Schottky contacts to $\mathrm{Nb}$-doped $\mathrm{SrTiO}_{3}$, where the insertion of an interfacial dipole via $\mathrm{LaAlO}_{3}(\mathrm{LAO})$ atomic layers sharply reduces the Schottky barrier height, yielding effectively Ohmic behavior at room temperature [20]. Such control over the charge-transport regimes across the interface offers an experimental platform to test the spin accumulation models.

Here, we examine charge and spin transport across $\mathrm{Co} / \mathrm{LaAlO}_{3} / \mathrm{Nb}: \mathrm{SrTiO}_{3}$ heterojunctions in the 3T geometry, where epitaxial layers of the wide-gap insulator $\mathrm{LaAlO}_{3}$ provide an interfacial dipole. Insertion of two unit cells (u.c.) of $\mathrm{LaAlO}_{3}$ decreases the contact resistance $\left(R_{C}\right)$ over 3 orders of magnitude, a signature of the internal electric field in the $\mathrm{LaAlO}_{3}$ barrier. We observe Lorentzian MR only for finite barrier thickness $\left(1<t_{\mathrm{LAO}}<4\right.$ u.c.), demonstrating a clear dependence on the presence of the oxide tunnel barrier and eliminating the possibility that the signals arise due to spin accumulation in either the semiconductor or in ILS. Further, through magnetic-field-dependent inelastic electron tunneling spectroscopy (IETS) measurements, we are able to determine that spin-dependent transport through defect states in the $\mathrm{LaAlO}_{3}$ barrier is responsible for the junction MR. We present a general hopping mechanism that can explain the magnetoresistance amplitude, linewidth, and anisotropy, all within a single analytic framework.

\section{SAMPLE FABRICATION}

We fabricate the structure shown in Fig. 1(a), consisting of cobalt on top of $200 \mathrm{~nm} 0.05$ wt \% $\mathrm{Nb}: \mathrm{SrTiO}_{3}$ with 0-5 u.c. epitaxial $\mathrm{LaAlO}_{3}$ interfacial barriers, forming an FM-I-NM heterostructure. These heterostructures are synthesized by a combination of pulsed laser deposition and $e$-beam deposition. Single-crystal $\mathrm{SrTiO}_{3}(001)$ substrates are preannealed in $1 \times 10^{-5}$ Torr of molecular oxygen at $900^{\circ} \mathrm{C}$ for $30 \mathrm{~min}$. Next, $200 \mathrm{~nm}$ of $0.05 \mathrm{wt} \% \mathrm{Nb}: \mathrm{SrTiO}_{3}$ is deposited by pulsed laser deposition $(\mathrm{KrF}, \lambda=248 \mathrm{~nm})$ at $1080^{\circ} \mathrm{C}$ under ultrahigh-vacuum conditions with a base pressure of $P=2 \times 10^{-9}$ Torr and a laser fluence of

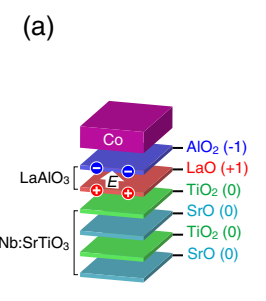

(b)


FIG. 1. Charge and spin transport across $\mathrm{Co} / \mathrm{LaAlO}_{3} /$ $\mathrm{Nb}: \mathrm{SrTiO}_{3}$ heterojunctions with interface dipole layers. (a) Schematic diagram for stacking of 1 u.c. $\mathrm{LaAlO}_{3}(001)$ inserted between $\mathrm{Co}$ and $\mathrm{Nb}: \mathrm{SrTiO}_{3}(001)$. (b) Schematic diagram of the measurement configuration. (c) $I_{C}$ vs $V_{C}$ for different $t_{\mathrm{LAO}}$. (d) Typical junction dc magnetoresistance $\left(t_{\mathrm{LAO}}=2\right.$ u.c., $\left.I_{C}=2.8 \mathrm{~mA}\right)$ as a function of $B^{\perp}$ (black) and $B^{\|}$(blue). Dashed lines indicate the Lorentzian component, while solid lines show the best fit including the background. (e) Bias dependence of the total Lorentzian MR amplitude $\left(\Delta V_{C}=\Delta V_{C}^{\perp}-\Delta V_{C}^{\|}\right)$for $t_{\mathrm{LAO}}=2$ u.c.

$0.43 \mathrm{~J} / \mathrm{cm}^{2}$. This promotes a step-flow growth mode and minimizes Sr vacancy formation [17]. The films are postannealed in $\mathrm{O}_{2}$ at $900^{\circ} \mathrm{C}$ at $P=1 \times 10^{-2}$ Torr for $30 \mathrm{~min}$. Typically, these films exhibit carrier densities near $1.6 \times 10^{19} \mathrm{~cm}^{-3}$, indicating full $\mathrm{Nb}$ activation, and Hall mobilities exceeding $1000 \mathrm{~cm}^{2} / \mathrm{Vs}$ at cryogenic temperatures. $\mathrm{LaAlO}_{3}$ is deposited at $650{ }^{\circ} \mathrm{C}\left(P=1 \times 10^{-6}\right.$ Torr $\mathrm{O}_{2}$ ) with a fluence of $0.45 \mathrm{~J} / \mathrm{cm}^{2}$, and the thickness is monitored by intensity oscillations in the reflection highenergy electron diffraction pattern. Before removing from the vacuum chamber, the heterostructure is postannealed to refill oxygen vacancies at $400{ }^{\circ} \mathrm{C}\left(P=360\right.$ Torr $\left.\mathrm{O}_{2}\right)$ for $40 \mathrm{~min}$. The resulting $\mathrm{LaAlO}_{3}$ surface morphology is atomically flat with terraces separated by several hundred nanometers, arising from the small miscut angle as typical for $\mathrm{SrTiO}_{3}(001)$ stepand-terrace substrates. The complex oxide structure is transferred ex situ to a metal evaporator. Prior to cobalt deposition at room temperature, the oxide heterostructures are annealed at $500^{\circ} \mathrm{C}\left(P=1 \times 10^{-5}\right.$ Torr $\left.\mathrm{O}_{2}\right)$ for $30 \mathrm{~min}$ [21]. The cobalt electrodes are patterned using an in situ shadow mask. Contacts to the cobalt pads are made by Ag painting with $\mathrm{Au}$ wire, and $\mathrm{Ohmic}$ contacts to the $\mathrm{Nb}: \mathrm{SrTiO}_{3}$ film are made by ultrasonic wire bonding with $\mathrm{Al}$ wire.

\section{EXPERIMENTAL RESULTS}

\section{A. Junction magnetoresistance in the 3T geometry}

$\mathrm{Co} / \mathrm{LaAlO}_{3} / \mathrm{Nb}: \mathrm{SrTiO}_{3} / \mathrm{SrTiO}_{3}(001)$ heterojunctions are characterized using the measurement setup shown in 
Fig. 1(b). All junctions exhibit nonlinear current-voltage $(I-V)$ characteristics as shown in Fig. 1(c). A clear transition from high resistance to high transmission is observed when $t_{\mathrm{LAO}}$ is tuned from 0 to 2 u.c. To probe the spin-dependent contribution to the junction transport, we apply fixed current $\left(I_{C}\right)$ and measure the heterojunction voltage $\left(V_{C}\right)$ for out-of-plane and inplane applied magnetic fields. A characteristic data set for a high transmission structure $\left(t_{\mathrm{LAO}}=2\right.$ u.c.) is shown in Fig. 1(d). The data are fit with the equation $V_{C}(B)=\Delta V_{C}^{\perp, \|} /\left[1+(B / \Delta B)^{2}\right]+f(B)$, where $B$ is the magnitude of the applied field, $\Delta V_{C}^{\perp, \|}$ is the anisotropic Lorentzian amplitude [positive for out-of-plane $\left(B^{\perp}\right)$ and negative for in-plane $\left(B^{\|}\right)$fields], and $f(B)$ is the background magnetoresistance. We use quadratic $f(B) \propto B^{2}$ for $B^{\perp}$ and linear $f(B) \propto|B|$ for $B^{\|}$. At higher fields $(B>2 \mathrm{~T})$, both field orientations exhibit linear dependence, up to measured fields of $10 \mathrm{~T}$. From the anisotropic contributions, we obtain the total Lorentzian MR $\Delta V_{C}=\Delta V_{C}^{\perp}-\Delta V_{C}^{\|}$. We note that for standard Hanle dephasing, an in-plane signal is not expected [5,22,23]. $\Delta V_{C}$ is plotted as a function of the bias voltage in Fig. 1(e), which shows a clear turn-on around $V_{C}=0.2 \mathrm{~V}$ and then increases approximately linearly. Although $\Delta V_{C}$ is small relative to $V_{C}\left(\Delta V_{C} / V_{C} \approx 5 \times 10^{-4}\right)$, it far exceeds the expected signal generated from a true spin accumulation in the semiconductor [2,24]. For instance, the measured "spin- $R A$ " $=\Delta V_{\mathrm{C}} A / I_{C}=1 \times 10^{4} \Omega \mu \mathrm{m}^{2} \gg$ intrinsic "spin- $R A "=\rho_{\mathrm{Nb}: \mathrm{STO}} \lambda_{s f}^{2} / t_{\mathrm{Nb}: \mathrm{STO}} \approx 6 \Omega \mu \mathrm{m}^{2}$, where $A$ is the junction area, $\rho_{\mathrm{Nb}: \text { STO }}$ is the $\mathrm{Nb}: \mathrm{SrTiO}_{3}$ resistivity at $T=10 \mathrm{~K}$, and we assume a spin diffusion length $\lambda_{s f}=1 \mu \mathrm{m}$. The linewidth $(\Delta B)$ is independent of applied bias with an average of $112 \pm 4 \mathrm{mT}$. In a 3T Hanle picture, this would correspond to a spin-dephasing time of $\tau_{s}=\hbar /\left(g \mu_{B} \Delta B\right) \approx 50 \mathrm{ps}$, where $g=2$ is the electron $g$ factor, $\mu_{B}$ is the Bohr magneton, and $\hbar$ is the reduced Planck constant.

Next, we discuss the effect of the interface dipole, varied as a function of $t_{\mathrm{LAO}}$, on the junction transport. Figure 2(a) (top panel, left axis) displays the zero-bias contact resistance-area product $\left(R_{C} A\right)$, exhibiting a minimum at $t_{\mathrm{LAO}}=$ 2 u.c. The dramatic decrease in $R_{C} A$ arises from the shifting of band alignments between the $\mathrm{Co}$ and $\mathrm{Nb}: \mathrm{SrTiO}_{3}$ by the $\mathrm{LaAlO}_{3}$ interface dipole [20]. From $t_{\mathrm{LAO}}=0$ to 2 u.c., there is a transition from the Schottky limit to nearly Ohmic behavior, limited by a small tunneling contribution at $t_{\mathrm{LAO}}=2$ u.c. Beyond 2 u.c., the depletion width is suppressed [20], but the contact resistance increases due to tunneling through the thicker barrier. The spin-dependent contribution $\left(\Delta V_{C}\right)$ is also plotted for different $t_{\text {LAO }}$ in Fig. 2(a) (top panel, right axis) for the same bias window $\left(V_{C}=0.4 \mathrm{~V}\right)$. We note that no signal is observed without the presence of an oxide barrier, consistent with previous reports $[3,4,9,25] ; \Delta V_{\mathrm{C}}$ turns on for
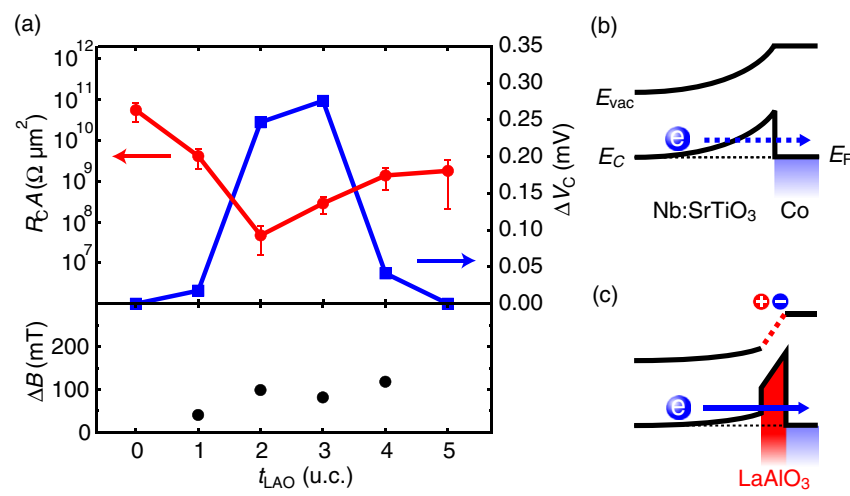

FIG. 2. Tuning charge and spin transport via artificial dipoles. (a) Top panel: Comparison of the junction zero-bias contact resistance-area product $\left(R_{C} A\right)$ (left axis, red circles) with $\Delta V_{\mathrm{C}}$ (right axis, blue squares) measured at $V_{C}=0.4 \mathrm{~V}$ as a function of $t_{\mathrm{LAO}}$. Bottom panel: Linewidths of the Lorentzian MR. (b),(c) Interface energy band diagrams illustrating the decrease of the Schottky barrier height and depletion width by the insertion of an interface dipole in the form of a single unit cell of $\mathrm{LaAlO}_{3}(001)$.

$t_{\text {LAO }}>0$ but subsequently decreases when $t_{\text {LAO }}>3$ u.c. The corresponding linewidths, in the range of $50-120 \mathrm{mT}$, are shown in the bottom panel of Fig. 2(a). The anticorrelation between charge and spin transport indicates that the barrier itself is critical to observation of spin-dependent behavior. Further, a decrease of the Schottky barrier height is expected to equilibrate any spin accumulations in the semiconductor and ILS, causing a reduction in $\Delta V_{C}$ $[14,21]$. We observe the opposite trend, which precludes spin accumulation in interfacial localized states as the origin.

\section{B. Spin-dependent tunneling spectroscopy}

The 3T geometry purposely isolates the junction in order to measure small changes in the junction voltage that might arise due to spin accumulation. However, since the current flows through the same junction, the measurement is inherently susceptible to inelastic contributions to the tunneling current. One way to parse out the different contributions is to examine them directly through IETS, which can be sensitive to defects, vibrational modes, spin waves, and trap states $[26,27]$. We perform magnetic-fielddependent IETS, in which an ac + dc voltage is applied and the dc, first, and second harmonics of the current and voltage response are measured to obtain $d^{2} i / d v^{2}$ [21]. If the Lorentzian MR arises from modulation of transport through the tunnel barrier, either by resonant or inelastic events, then it should be evident in the field-modulated IETS response [28]. Figure 3(a) shows $d^{2} i / d v^{2}$ as a function of magnetic field for $t_{\mathrm{LAO}}=2$ and 3 u.c., where the Lorentzian MR is evident, with similar line shapes, widths, and $B^{\perp}$ vs $B^{\|}$anisotropy compared with the observed dc response [see Fig. 1(d)], demonstrating that they have the same underlying origin. The sign inversion is 




(b)

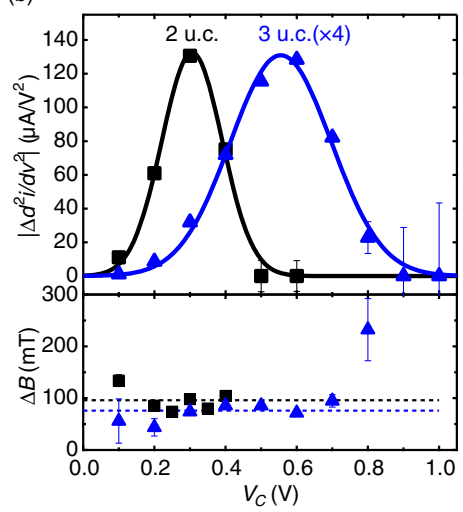

FIG. 3. Magnetic-field-modulated inelastic electron tunneling spectroscopy. (a) Magnetic field dependence of $d^{2} i / d v^{2}$ for $t_{\mathrm{LAO}}=2$ and 3 u.c. for $B^{\perp}$ (black circles) and $B^{\|}$(red circles). The bias state for 2 u.c. is $V_{C}=0.3 \mathrm{~V}, d v=50 \mathrm{mV}$, and $V_{C}=0.4 \mathrm{~V}, d v=50 \mathrm{mV}$ for 3 u.c. (b) Top panel: $\left|\Delta d^{2} i / d v^{2}\right|$ for the 2 u.c. (black squares) and 3 u.c. (blue triangles) $\mathrm{LaAlO}_{3}$ tunnel barriers. Measured at fixed ac voltage, $d v=50 \mathrm{mV}$ and $T=5 \mathrm{~K}$. Bottom panel: Corresponding linewidths of the total Lorentzian contribution extracted from $d^{2} i / d v^{2}$.

expected because $d^{2} i / d v^{2}$ is a measured current response under fixed applied voltage, while $\Delta V_{C}$ is a voltage response under fixed $I_{C}$. The total Lorentzian contribution to the IETS MR $\left(\Delta d^{2} i / d v^{2}\right)$ is extracted similar to the dc fitting procedure in Fig. 1(d) and is only a small change relative to the field-independent background $\left(\Delta d^{2} i / d v^{2}\right) /$ $\left(d^{2} i / d v^{2}\right) \leq 1 \times 10^{-3}$. Because of the noise levels and small signals, we are unable to detect the junction MR in the second harmonic for $t_{\mathrm{LAO}}=1$ and 4 u.c.

The spin-dependent transport observed in IETS depends strongly on the bias voltage as well as $t_{\mathrm{LAO}}$. This is demonstrated in the top panel of Fig. 3(b), which plots $\left|\Delta d^{2} i / d v^{2}\right|$ as a function of the dc bias $V_{C}$. For $t_{\mathrm{LAO}}=2$ and 3 u.c., there is a gradual turn-on in the magnetic-fieldmodulated response of $d^{2} i / d v^{2}$ at low bias, followed by a maximum in $\left|\Delta d^{2} i / d v^{2}\right|$, and a subsequent decrease at higher bias. The bias dependence can be described by a Gaussian distribution $\left|\Delta d^{2} i / d v^{2}\right| \propto e^{-\left(E-E_{0}\right)^{2} / \Delta E^{2}}$, with $E_{0}=305 \mathrm{meV}, \Delta E=120 \mathrm{meV}$ and $E_{0}=555 \mathrm{meV}$, $\Delta E=200 \mathrm{meV}$, for $t_{\mathrm{LAO}}=2$ and 3 u.c., respectively. Such a distribution is further evidence that the origin involves transport through defect states in the barrier, as opposed to spin-dependent changes in the band structure [21]. The relatively broad energy dependence, which could involve either coherent or incoherent tunneling processes, accounts for the dc dependence of $\Delta V_{C}$ through a double integration. The linewidth [Fig. 3(b), bottom panel] does not vary across the spectrum but does correspond identically with the dc linewidths in Fig. 2(a) (bottom panel). Lastly, we note a clear shift in $E_{0}$ from 2 to 3 u.c. $\mathrm{LaAlO}_{3}$, stemming from the spontaneous electric field in the barrier and demonstrating interfacial polarization control over spin-dependent transport in oxide heterostructures.

\section{SPIN-DEPENDENT HOPPING MODEL}

These experiments rule out spin accumulation as the origin of the junction MR. Alternatively, a spin-blockade mechanism involving resonant tunneling through defect states has been recently proposed [12,29]. Very broadly, spin-dependent hopping is a general phenomenon involving transport across localized states and does not apply solely to these structures [30-35]. A single impurity can cause a spin blockade if it is located in between FM and $\mathrm{NM}$ electrodes as shown in Fig. 4(a) for the case of $\mathrm{Co} / \mathrm{LaAlO}_{3} / \mathrm{Nb}: \mathrm{SrTiO}_{3}$. Hopping via the defect is restricted if the electrons have antiparallel spin alignment relative to the FM or if the defect state is already occupied. However, if the state is acted on by either an external field $(B)$ or by local fields $\left(B_{L}\right)$, then the defect spin can evolve with time, enforcing or removing the blocking effect depending on the amplitude of $B$ relative to $B_{L}$ and the angle $(\theta)$ between the total field $\left(\boldsymbol{B}_{T}=\boldsymbol{B}+\boldsymbol{B}_{L}\right)$ and the FM magnetization $(\boldsymbol{M})$ (fixed in plane for $B<2 \mathrm{~T}$ ). (a)

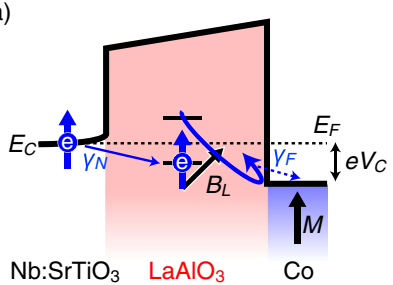

(c)



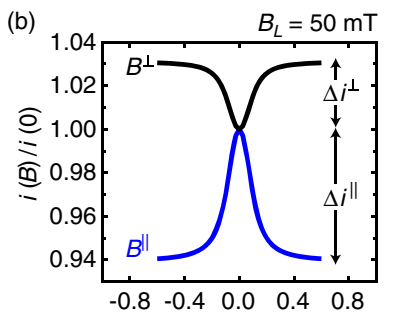

(d)

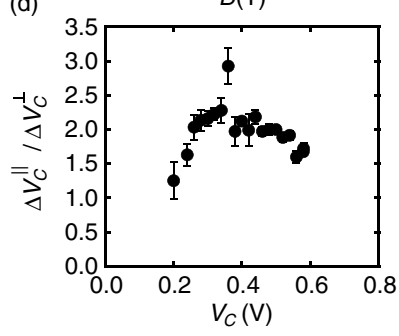

FIG. 4. Spin-dependent hopping as the origin for junction magnetoresistance. (a) Schematic diagram for electron hopping through a single defect between a FM and NM contact in the electron-extraction regime. The schematic is drawn for optimally dipole tuned $\mathrm{Co} / \mathrm{LaAlO}_{3} / \mathrm{Nb}: \mathrm{SrTiO}_{3}$ near the flat-band condition. (b) Calculated magnetic field dependence of the hopping current through a localized state. The linewidth is determined by the local fields acting upon the defect site, here plotted for $B_{L}=50 \mathrm{mT} \approx \Delta B / 2$, which reproduces the experimental linewidths. (c) Dependence of the anisotropic components $\Delta i^{\perp, \|}$ on the tunnel coupling rate $\left(\gamma_{F}\right)$ normalized to $\omega_{L}=g \mu_{B} B_{L} / \hbar$. Solid lines represent $B^{\|}$and dashed lines $B^{\perp}$ at different values of the coupling $\left(\gamma_{N}\right)$ between the defect state and the $\mathrm{Nb}: \mathrm{SrTiO}_{3}$. (d) Measured anisotropy $\Delta V_{C}^{\|} / \Delta V_{C}^{\perp}$ as a function of bias for $t_{\mathrm{LAO}}=2$ u.c. 
In the steady state, the rate equation for the evolution of the defect-state population $(p)$ is

$$
\dot{p}=-\frac{1}{2}\left(1+P_{d} P_{F}\right) \gamma_{F} p+\gamma_{N}(1-p)=0,
$$

where $P_{F}$ is the Fermi-level spin polarization of the FM, and $\gamma_{F}$ and $\gamma_{N}$ are the rates of tunneling between the defect state and the FM or NM, respectively. We consider only the extraction regime here (the current is running from NM to FM). Hopping from NM to the defect site occurs with rate $\gamma_{N}$ while hopping from the defect to FM occurs at the rate $\frac{1}{2}\left(1+P_{d} P_{F}\right) \gamma_{F}$. The prefactor involving the spin polarization of the defect state $\left(P_{d}\right)$ and FM Fermi-level density of states $\left(P_{F}\right)$ describes the probability for the defect spin to be parallel to the FM magnetization. In the ideal case of a half-metal $\left(P_{F}=1\right)$, only parallel defect spins are permitted to travel to the FM. Antiparallel spins are effectively blocked by the Fermi-level spin polarization of the FM, which serves as a spin analyzer, causing the defect sites to develop a net spin polarization $-P_{F}$. However, the presence of a magnetic field rotates these blockaded spins. When the defect is quickly refilled after a spin vacates it, the timeaveraged defect polarization is

$$
\begin{aligned}
P_{d} & =-\gamma_{F} \int_{0}^{\infty} P_{F}\left(\cos \omega_{T} t \sin ^{2} \theta+\cos ^{2} \theta\right) e^{-\gamma_{F} t} d t \\
& =-P_{F} \frac{\gamma_{F}^{2}+\omega_{T}^{2} \cos ^{2} \theta}{\gamma_{F}^{2}+\omega_{T}^{2}}=-P_{F} \chi\left(\boldsymbol{B}_{T}\right),
\end{aligned}
$$

where $\omega_{T}$ is the magnitude of the total magnetic field in units of frequency and $\theta$ is the angle that the total field makes with the FM magnetization.

After taking into account the field-sensitive polarization of the defect spin, Eq. (1) reads

$$
\dot{p}=-\frac{1}{2}\left[1-P_{F}^{2} \chi\left(\boldsymbol{B}_{T}\right)\right] \gamma_{F} p+\gamma_{N}(1-p)=0 .
$$

The tunneling current in the steady state is $i=$ $\left\langle(e / 2)\left[1-P_{F}^{2} \chi\left(\boldsymbol{B}_{T}\right)\right] \gamma_{F} p\right\rangle$, which yields the spindependent hopping current:

$$
i=\left\langle\frac{e\left[1-P_{F}^{2} \chi\left(\boldsymbol{B}_{T}\right)\right] \gamma_{F} \gamma_{N}}{\left[1-P_{F}^{2} \chi\left(\boldsymbol{B}_{T}\right)\right] \gamma_{F}+2 \gamma_{N}}\right\rangle .
$$

Here, we average over an isotropic Gaussian distribution for the local field, which is denoted by the angular brackets. The final result of Eq. (4) from our general formulation agrees with the calculation of resonant tunneling current by Ref. [12] as expected for tunneling through a single level [36]. The anisotropy between parallel and transverse field orientations is a key prediction of the hopping model: $B^{\|}$ inhibits the ability of the local field to change the spin orientation, and thereby the current is reduced, while $B^{\perp}$ precesses the defect spin, unblocking the transport channel. This is a general formulation that does not rely on the underlying tunneling process (i.e., resonant, inelastic, or sequential hopping).

Within this framework, the signal linewidth, amplitude, and anisotropy arise naturally. Figure 4(b) shows the calculated field-modulated hopping $i(B) / i(0)$, which reproduces the experimental data [21]. Considering Eq. (2), we can say that the transport is in the slow hopping regime $\left(\omega_{L}>\gamma_{F}\right.$, where $\omega_{L}$ is the precession due to $\left.B_{L}\right)$. $B_{L}$, which determines the Lorentzian linewidth, can arise from exchange, hyperfine, and spin-orbit interactions. Experimentally, $\Delta B$ is similar to estimated hyperfine fields of ${ }^{27} \mathrm{Al}$ and ${ }^{139} \mathrm{La}$ nuclear moments of approximately 100 $200 \mathrm{mT}$ [3], but spin-orbit fields and anisotropic $g$ factors should also be considered [37,38]. Figure 4(c) plots the predicted anisotropy between $\Delta i^{\|}$and $\Delta i^{\perp}$ as a function of $\gamma_{F}$. In the slow hopping regime $\left(\gamma_{F}<\omega_{L}\right)$, the model predicts that the amplitude of the in-plane MR should exceed the out-of-plane signal, consistent with these and other experimental observations [1,5,9,22]. As can be seen from the limiting case $\gamma_{F} \ll \omega_{L}$, an anisotropy ratio

$$
\frac{\Delta i^{\|}}{\Delta i^{\perp}} \approx 1-\frac{1}{\left\langle\cos ^{2} \theta_{L}\right\rangle}=-2
$$

is expected, which agrees well with the measured anisotropy ratio for 2 u.c. $\mathrm{LaAlO}_{3}$ shown in Fig. 4(d) (note that $\Delta V_{C}^{\|} / \Delta V_{C}^{\perp}=\Delta i^{\|} / \Delta i^{\perp}$ ). The negative sign comes from the opposite responses of the current in the parallel and perpendicular field configurations. Finally, considering defect hopping in parallel with direct tunneling, we can estimate an areal and energy density of defect states $D \approx 3 \times 10^{9} \mathrm{eV}^{-1} \mathrm{~cm}^{-2}$ [21]. Thus, the measurement can probe relatively low defect densities and is sensitive to the evolution of a single spin state involved in the junction transport.

\section{CONCLUSION}

In summary, we have examined the problem of the junction $\mathrm{MR}$ in $\mathrm{SrTiO}_{3}$-based heterojunctions by taking advantage of the built-in electric field in atomically thin layers of $\mathrm{LaAlO}_{3}$. Control over band alignments through artificial interfacial dipoles is a highly effective method for reducing the Schottky barrier and provides an experimental route for manipulating charge and spin transport across the heterostructure. We have demonstrated that the Lorentzian MR observed in $\mathrm{Co} / \mathrm{LaAlO}_{3} / \mathrm{Nb}: \mathrm{SrTiO}_{3}$ tunnel junctions is inconsistent with Hanle dephasing of spin accumulation in either the semiconductor or in ILS. Instead, spindependent hopping through defect states successfully accounts for the observed behavior. This phenomenon, which involves only a single defect state in the transport process, is highly sensitive to low defect densities and 
presents a new spin-sensitive approach for examining local magnetic fields in solid-state heterostructures.

\section{ACKNOWLEDGMENTS}

A. G. S., H. I., and H. Y.H. acknowledge device fabrication and magnetotransport experiments from Function Accelerated nanoMaterial Engineering (FAME), one of six centers of Semiconductor Technology Advanced Research network (STARnet), a Semiconductor Research Corporation (SRC) program sponsored by Microelectronics Advanced Research Corporation (MARCO) and Defense Advanced Research Projects Agency (DARPA). T. T. and Y. H. acknowledge support for materials synthesis from the U.S. Department of Energy, Office of Basic Energy Sciences, Division of Materials Sciences and Engineering, under Contract No. DE-AC02-76SF00515. N. J. H. and M. E. F. acknowledge support by The Center for Spintronic Materials, Interfaces, and Novel Architectures (C-SPIN), one of six centers of STARnet, a Semiconductor Research Corporation program, sponsored by MARCO and DARPA.

H. I. and A. G. S. contributed equally to this work.

[1] S. P. Dash, S. Sharma, R. S. Patel, M. P. de Jong, and R. Jansen, Electrical Creation of Spin Polarization in Silicon at Room Temperature, Nature (London) 462, 491 (2009).

[2] M. Tran, H. Jaffrès, C. Deranlot, J.-M. George, A. Fert, A. Miard, and A. Lemaître, Enhancement of the Spin Accumulation at the Interface between a Spin-Polarized Tunnel Junction and a Semiconductor, Phys. Rev. Lett. 102, 036601 (2009).

[3] N. Reyren, M. Bibes, E. Lesne, J.-M. George, C. Deranlot, S. Collin, A. Barthélémy, and H. Jaffrès, Gate-Controlled Spin Injection at $\mathrm{LaAlO}_{3} / \mathrm{SrTiO}_{3}$ Interfaces, Phys. Rev. Lett. 108, 186802 (2012).

[4] W. Han, X. Jiang, A. Kajdos, S.-H. Yang, S. Stemmer, and S. S. P. Parkin, Spin Injection and Detection in Lanthanumand Niobium-Doped $\mathrm{SrTiO}_{3}$ Using the Hanle Technique, Nat. Commun. 4, 2134 (2013).

[5] R. Jansen, S. P. Dash, S. Sharma, and B. C. Min, Silicon Spintronics with Ferromagnetic Tunnel Devices, Semicond. Sci. Technol. 27, 083001 (2012).

[6] X. Lou, C. Adelmann, M. Furis, S. A. Crooker, C. J. Palmstrøm, and P. A. Crowell, Electrical Detection of Spin Accumulation at a Ferromagnet-Semiconductor Interface, Phys. Rev. Lett. 96, 176603 (2006).

[7] O. Txoperena, M. Gobbi, A. Bedoya-Pinto, F. Golmar, X. Sun, L. E. Hueso, and F. Casanova, How Reliable Are Hanle Measurements in Metals in a Three-Terminal Geometry? Appl. Phys. Lett. 102, 192406 (2013).

[8] O. Txoperena, Y. Song, L. Qing, M. Gobbi, L. E. Hueso, H. Dery, and F. Casanova, Impurity-Assisted Tunneling Magnetoresistance under a Weak Magnetic Field, Phys. Rev. Lett. 113, 146601 (2014).

[9] A. G. Swartz, S. Harashima, Y. Xie, D. Lu, B. Kim, C. Bell, Y. Hikita, and H. Y. Hwang, Spin-Dependent Transport across $\mathrm{Co} / \mathrm{LaAlO}_{3} / \mathrm{SrTiO}_{3}$ Heterojunctions, Appl. Phys. Lett. 105, 032406 (2014).

[10] R. I. Dzhioev, K. V. Kavokin, V. L. Korenev, M. V. Lazarev, B. Ya. Meltser, M. N. Stepanova, B. P. Zakharchenya, D. Gammon, and D. S. Katzer, Low-Temperature Spin Relaxation in n-Type GaAs, Phys. Rev. B 66, 245204 (2002).

[11] J. M. Kikkawa and D. D. Awschalom, Resonant Spin Amplification in n-Type GaAs, Phys. Rev. Lett. 80, 4313 (1998).

[12] Y. Song and H. Dery, Magnetic-Field-Modulated Resonant Tunneling in Ferromagnetic-Insulator-Nonmagnetic Junctions, Phys. Rev. Lett. 113, 047205 (2014).

[13] S. G. Bhat and P. S. A. Kumar, Room Temperature Electrical Spin Injection into GaAs by an Oxide Spin Injector, Sci. Rep. 4, 5588 (2014).

[14] R. Jansen, A. M. Deac, H. Saito, and S. Yuasa, Injection and Detection of Spin in a Semiconductor by Tunneling via Interface States, Phys. Rev. B 85, 134420 (2012).

[15] H. Y. Hwang, Y. Iwasa, M. Kawasaki, B. Keimer, N. Nagaosa, and Y. Tokura, Emergent Phenomena at Oxide Interfaces, Nat. Mater. 11, 103 (2012).

[16] O. N. Tufte and P.W. Chapman, Electron Mobility in Semiconducting Strontium Titanate, Phys. Rev. 155, 796 (1967).

[17] Y. Kozuka, Y. Hikita, C. Bell, and H. Y. Hwang, Dramatic Mobility Enhancements in Doped $\mathrm{SrTiO}_{3}$ Thin Films by Defect Management, Appl. Phys. Lett. 97, 012107 (2010).

[18] C. Şahin, G. Vignale, and M. E. Flatté, Derivation of Effective Spin-Orbit Hamiltonians and Spin Lifetimes, with Application to $\mathrm{SrTiO}_{3}$ Heterostructures, Phys. Rev. B 89, 155402 (2014).

[19] A. M. Kamerbeek, P. Högl, J. Fabian, and T. Banerjee, Electric Field Control of Spin Lifetimes in $\mathrm{Nb}-\mathrm{SrTiO}_{3}$ by Spin-Orbit Fields, Phys. Rev. Lett. 115, 136601 (2015).

[20] T. Yajima, M. Minohara, C. Bell, H. Kumigashira, M. Oshima, H. Y. Hwang, and Y. Hikita, Enhanced Electrical Transparency by Ultrathin $\mathrm{LaAlO}_{3}$ Insertion at Oxide Metal/Semiconductor Heterointerfaces, Nano Lett. 15, 1622 (2015).

[21] See Supplemental Material at http://link.aps.org/ supplemental/10.1103/PhysRevX.5.041023 for the details on the contact synthesis, the principle of IETS, examinations of the spin accumulation and ILS models, and additional details on the spin-dependent hopping models.

[22] S. P. Dash, S. Sharma, J. C. Le Breton, J. Peiro, H. Jaffrès, J.-M. George, A. Lemaître, and R. Jansen, Spin Precession and Inverted Hanle Effect in a Semiconductor near a FiniteRoughness Ferromagnetic Interface, Phys. Rev. B 84, 054410 (2011).

[23] Y. Aoki, M. Kameno, Y. Ando, E. Shikoh, Y. Suzuki, T. Shinjo, M. Shiraishi, T. Sasaki, T. Oikawa, and T. Suzuki, Investigation of the Inverted Hanle Effect in Highly Doped Si, Phys. Rev. B 86, 081201 (2012).

[24] I. Appelbaum, H. N. Tinkey, and P. Li, Self-Consistent Model of Spin Accumulation Magnetoresistance in Ferromagnet/Insulator/Semiconductor Tunnel Junctions, Phys. Rev. B 90, 220402 (2014).

[25] A. M. Kamerbeek, E. K. de Vries, A. Dankert, S. P. Dash, B. J. van Wees, and T. Banerjee, Electric Field Effects on 
Spin Accumulation in Nb-Doped $\mathrm{SrTiO}_{3}$ Using Tunable Spin Injection Contacts at Room Temperature, Appl. Phys. Lett. 104, 212106 (2014).

[26] D. C. Tsui, R. E. Dietz, and L. R. Walker, Multiple Magnon Excitation in NiO by Electron Tunneling, Phys. Rev. Lett. 27, 1729 (1971).

[27] C. J. Adkins and W. A. Phillips, Inelastic Electron Tunnelling Spectroscopy, J. Phys. C 18, 1313 (1985).

[28] H. N. Tinkey, P. Li, and I. Appelbaum, Inelastic Electron Tunneling Spectroscopy of Local "Spin Accumulation" Devices, Appl. Phys. Lett. 104, 232410 (2014).

[29] Z. Yue, M. C. Prestgard, A. Tiwari, and M. E. Raikh, Resonant Magnetotunneling between Normal and Ferromagnetic Electrodes in Relation to the Three-Terminal Spin Transport, Phys. Rev. B 91, 195316 (2015).

[30] K. Ono, D. G. Austing, Y. Tokura, and S. Tarucha, Current Rectification by Pauli Exclusion in a Weakly Coupled Double Quantum Dot System, Science 297, 1313 (2002).

[31] D. Ephron, Y. Xu, and M. R. Beasley, Observation of Coulomb Correlations of Resonant Tunneling and Inelastic Hopping, Phys. Rev. Lett. 69, 3112 (1992).

[32] Ö. Mermer, G. Veeraraghavan, T. L. Francis, Y. Sheng, D. T. Nguyen, M. Wohlgenannt, A. Köhler, M. K. Al-Suti, and
M. S. Khan, Large Magnetoresistance in Nonmagnetic $\pi$ Conjugated Semiconductor Thin Film Devices, Phys. Rev. B 72, 205202 (2005).

[33] P. A. Bobbert, T. D. Nguyen, F. W. A. van Oost, B. Koopmans, and M. Wohlgenannt, Bipolaron Mechanism for Organic Magnetoresistance, Phys. Rev. Lett. 99, 216801 (2007).

[34] N. J. Harmon and M. E. Flatté, Spin-Flip Induced Magnetoresistance in Positionally Disordered Organic Solids, Phys. Rev. Lett. 108, 186602 (2012).

[35] N. J. Harmon and M. E. Flatté, Organic Magnetoresistance from Deep Traps, J. Appl. Phys. 116, 043707 (2014).

[36] J. H. Davies, S. Hershfield, P. Hyldgaard, and J. W. Wilkins, Current and Rate Equation for Resonant Tunneling, Phys. Rev. B 47, 4603 (1993).

[37] Y. Sheng, T. D. Nguyen, G. Veeraraghavan, Ö. Mermer, and M. Wohlgenannt, Effect of Spin-Orbit Coupling on Magnetoresistance in Organic Semiconductors, Phys. Rev. B 75, 035202 (2007).

[38] P. Li, J. Li, L. Qing, H. Dery, and I. Appelbaum, AnisotropyDriven Spin Relaxation in Germanium, Phys. Rev. Lett. 111, 257204 (2013). 